\title{
Aorta Disease
}

National Cancer Institute

\section{Source}

National Cancer Institute. Aorta Disease. NCI Thesaurus. Code C101253.

Pathology involving the thoracic, thoracoabdominal, or abdominal aorta (including aneurysms). (ACC) 